\title{
Real-Time Ratiometric Imaging of Micelles Assembly State in a Microfluidic Cancer-on-a-Chip
}

Natalia Feiner-Gracia, ${ }^{\bigcirc}$ Adrianna Glinkowska Mares, ${ }^{\bigcirc}$ Marina Buzhor, Romen Rodriguez-Trujillo, Josep Samitier Marti, Roey J. Amir,* Silvia Pujals, and Lorenzo Albertazzi*

Cite This: ACS Appl. Bio Mater. 2021, 4, 669-681

Read Online

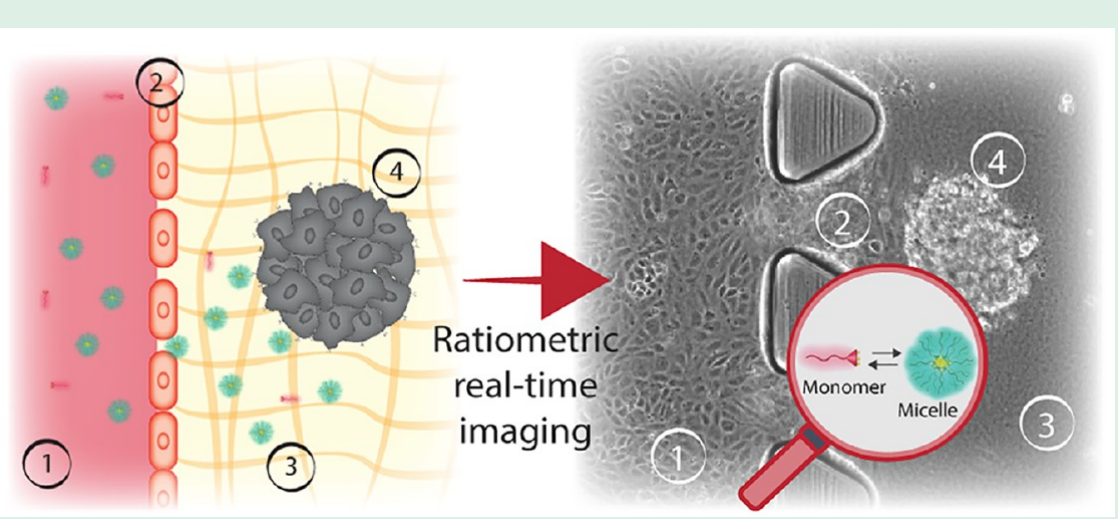

ABSTRACT: The performance of supramolecular nanocarriers as drug delivery systems depends on their stability in the complex and dynamic biological media. After administration, nanocarriers are challenged by physiological barriers such as shear stress and proteins present in blood, endothelial wall, extracellular matrix, and eventually cancer cell membrane. While early disassembly will result in a premature drug release, extreme stability of the nanocarriers can lead to poor drug release and low efficiency. Therefore, comprehensive understanding of the stability and assembly state of supramolecular carriers in each stage of delivery is the key factor for the rational design of these systems. One of the main challenges is that current $2 \mathrm{D}$ in vitro models do not provide exhaustive information, as they fail to recapitulate the $3 \mathrm{D}$ tumor microenvironment. This deficiency in the $2 \mathrm{D}$ model complexity is the main reason for the differences observed in vivo when testing the performance of supramolecular nanocarriers. Herein, we present a realtime monitoring study of self-assembled micelles stability and extravasation, combining spectral confocal microscopy and a microfluidic cancer-on-a-chip. The combination of advanced imaging and a reliable 3D model allows tracking of micelle disassembly by following the spectral properties of the amphiphiles in space and time during the crucial steps of drug delivery. The spectrally active micelles were introduced under flow and their position and conformation continuously followed by spectral imaging during the crossing of barriers, revealing the interplay between carrier structure, micellar stability, and extravasation. Integrating the ability of the micelles to change their fluorescent properties when disassembled, spectral confocal imaging and 3D microfluidic tumor blood vessel-on-a-chip resulted in the establishment of a robust testing platform suitable for real-time imaging and evaluation of supramolecular drug delivery carrier's stability.

KEYWORDS: micelle, stability, cancer-on-a-chip, supramolecular, nanoparticle, microfluidic

\section{INTRODUCTION}

Supramolecular nanocarriers, such as liposomes or micelles, are broadly investigated as potential drug delivery systems (DDS) for cancer therapy. ${ }^{1,2}$ Since Doxil, a liposome-encapsulated Doxorubicin, was approved in $1995,{ }^{3}$ the therapeutic efficacy of many nanosystems with different chemical features has been tested. However, low accumulation of the nanoparticles (NPs) in the solid tumor is still a major issue ${ }^{4}$ and one of the key reasons for failures in clinical trials. One of the main challenges with the use of supramolecular nanocarriers is to control their assembly-disassembly equilibrium, which determines in vivo success. The exposure to physiological environment affects the properties and decreases the number of fully assembled NPs arriving to the target site. ${ }^{5}$ Therefore, it is important to design nanosystems that are stable once injected into the body, and "smart" to free up the cargo when the target is reached.

Received: September 21, 2020

Accepted: December 1, 2020

Published: December 23, 2020 
$a$,

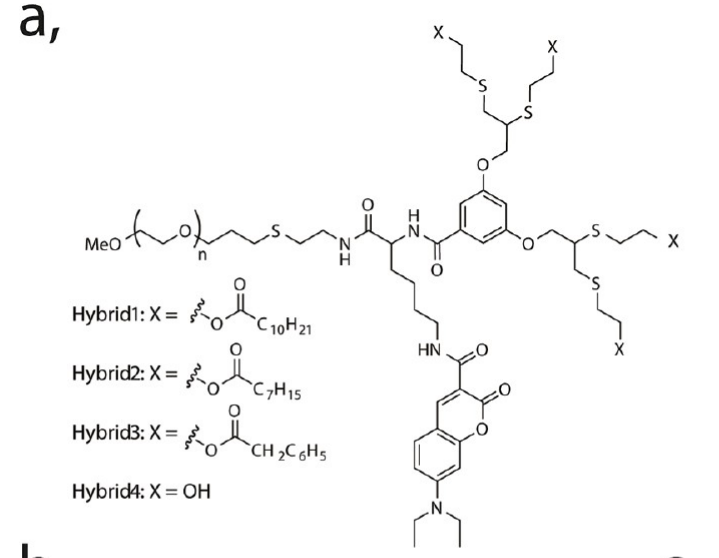

b,

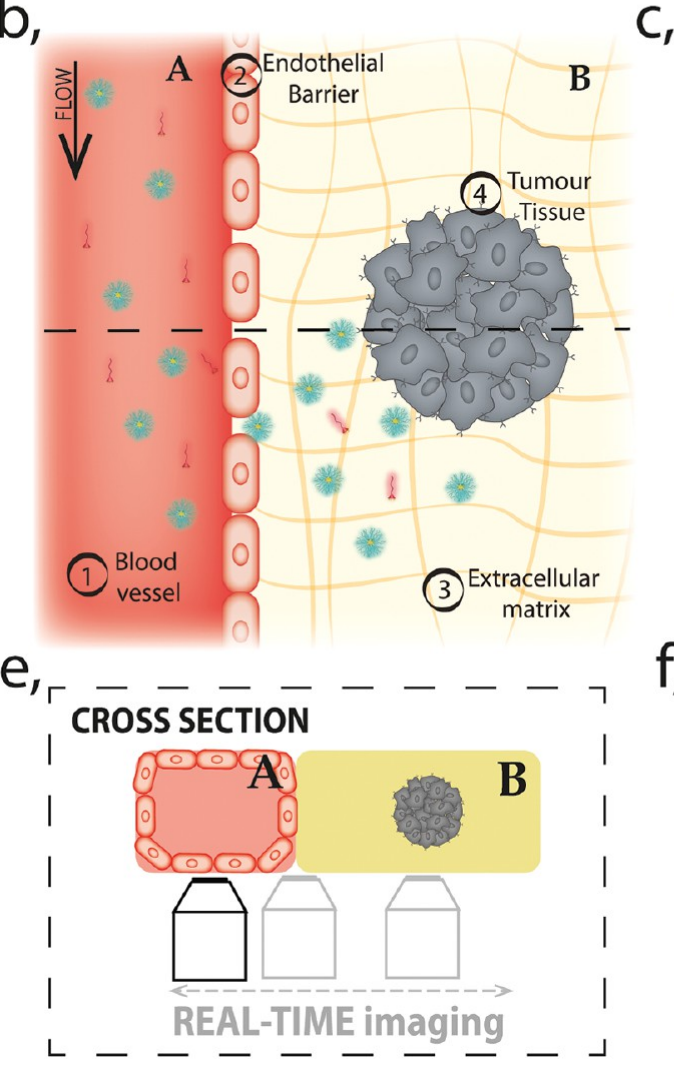

C,
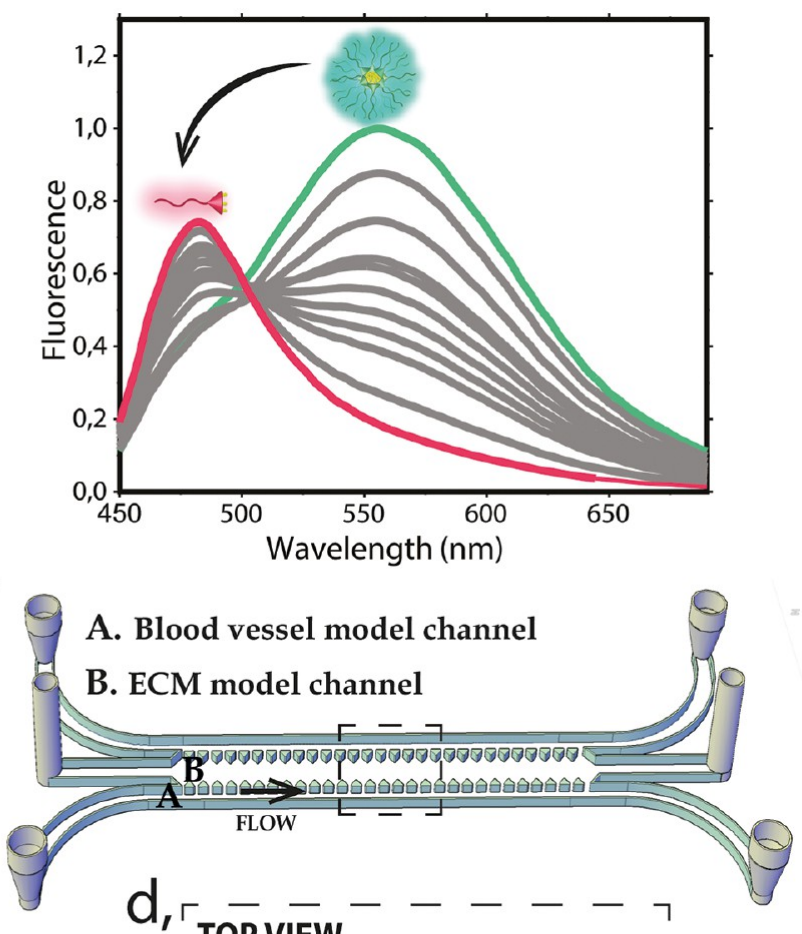

A. Blood vessel model channel

B. ECM model channel

Figure 1. Schematic representation of the model. (a) Molecular structure of the amphiphilic PEG-dendron hybrid polymers (left). Fluorescence emission graph for micelle-monomer equilibrium (right). In magenta, monomer (fully disassembled structure) and in green (fully assembled), micelle. (b) Schematic illustration of the top view of the model, with the reconstructed barriers marked as (1-4), two main regions (A, blood vessel channel and B, ECM model), and a cross-section indicated by dashed line for the projection of panel e. (c) 3D drawing of the microfluidic chip including inlets and outlets of each channel. A and B indicate two main compartments of the model: the blood vessel and ECM and the dashed-line marks area for the projection of the view in panel d. (d) Top view illustration of the microfluidic chip indicating the localization of the (A) blood vessel model channel and the (B) ECM model. The channel A is under continuous perfusion as schematically represented. (e) Cross-section illustration of the model (A and B, blood vessel/ECM model channels, respectively) and the scheme of the real-time imaging setup. (f) Zoom into the 3D representation (from panel c) showing the perspective of the channels before and after the complete model reconstruction. It illustrates how HUVECs line the blood vessel model channel covering the pillars and the collagen gel scaffold, forming a vertical endothelial barrier.

Right at the injection site, the NPs are subjected to a high dilution, interactions with serum proteins, and shear stress in a blood vessel, which favor their disassembly and lead to premature drug release into the bloodstream, causing systemic cytotoxicity or uncontrolled drug distribution. ${ }^{6-8}$ Internal walls of blood vessels are layered with endothelial cells (ECs), connected by characteristic tight junctions, which create a physical barrier, allowing the diffusion of only small molecules. ${ }^{9}$ Interactions with ECs membrane, upon NPs extravasation, can further compromise their stability. Nowadays, most of nano- particles-based DDS rely on the enhanced permeability and retention (EPR) effect. ${ }^{10}$ During EPR, the endothelial junctions are impaired, creating gaps that allow larger molecules to leave the systemic circulation, ${ }^{11}$ thus opening an escape route for the NPs. ${ }^{12}$ Particles able to extravasate through the "leaky" endothelial barrier (EB) arrive to the extracellular matrix (ECM) and the cancer cells. The ECM can differ in $\mathrm{pH}$ and shear stress comparing to the blood vessel, ${ }^{13}$ and its components can affect the NPs assembly equilibrium. ${ }^{5}$ The predictability and understanding of the stability of supramolecular assemblies 

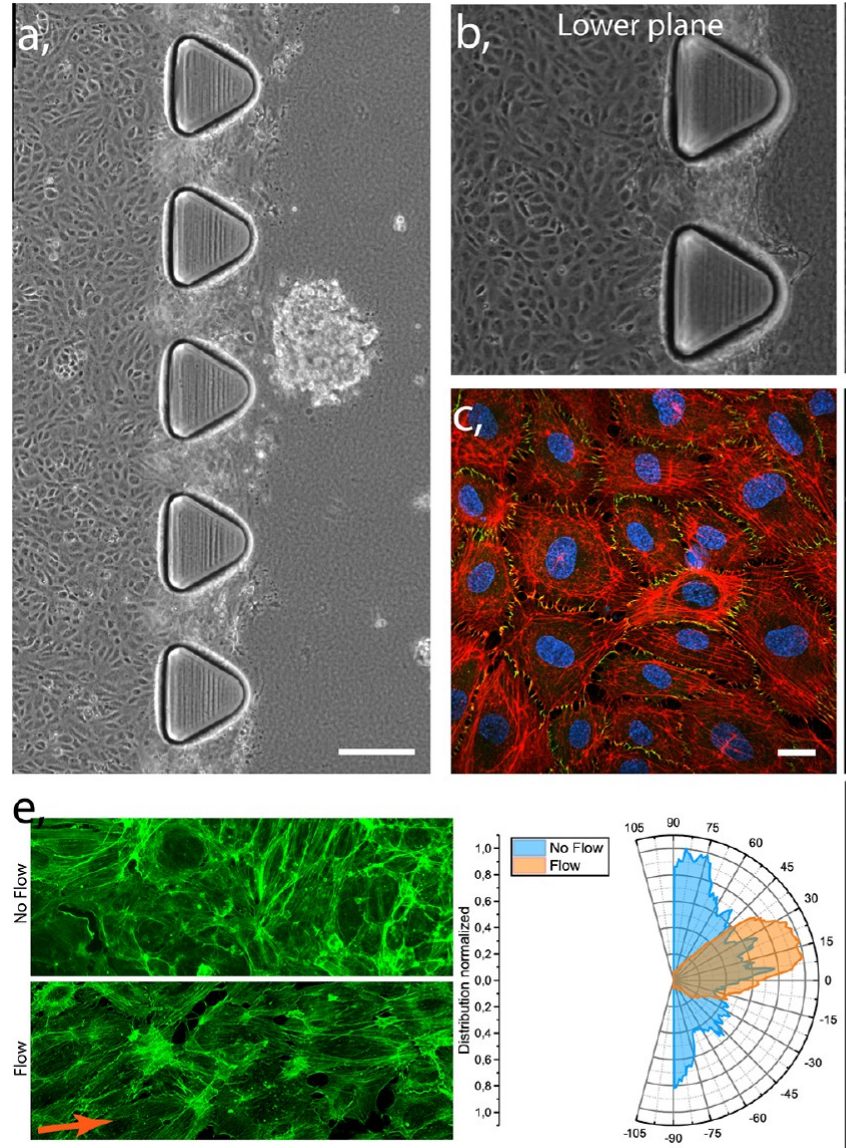
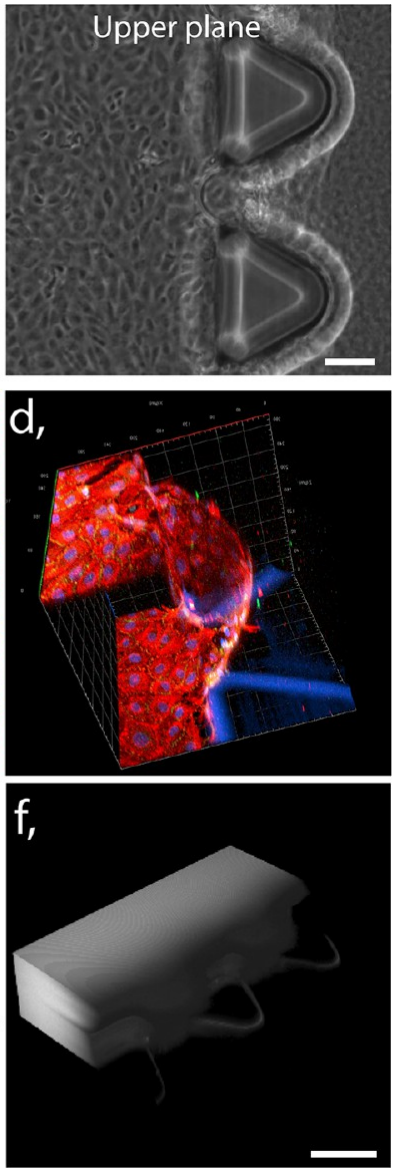

Figure 2. Functionality of the endothelial barrier model. (a) Transmission image showing complete tumor blood vessel-on-a-chip model with HUVECs lined lateral channel (left part of the chip) and adhering ECM model channel (right) with gel embedded HeLa spheroid. Scale bar $200 \mu$ m. (b) Transmission image of the lower and upper plane of the blood vessel model covered with confluent HUVECs monolayer. Scale bar $100 \mu \mathrm{m}$. (c) Confocal image of HUVECs confluent monolayer (red, actin; blue, nucleus; green, ZO-1). Scale bar $20 \mu \mathrm{m}$. (d) 3D reconstruction of confocal image of vertically grown HUVECs layer on the scaffold of collagen gel between the chip's microposts (red, actin; blue, nucleus; green, ZO-1); scale: axis ticks separation $40 \mu \mathrm{m}$, blue triangles represent base of the microposts. (e) Confocal images of actin stained (green) HUVECs in static (top) and perfused (bottom) blood vessel model channel and graph of actin filaments organization, demonstrating alignment under the flow and random orientation in static culture. (f) Epifluorescent microscopy 3D reconstructed image of continuously perfused $10 \mathrm{kDa}$ Dextran through the endothelialized blood vessel model channel at a time point $10 \mathrm{~min}$. Scale bar $150 \mu \mathrm{m}$.

within these changing conditions can pave the way to an improved design of nanosystems to be translated into the clinics.

Because of the difficulties in real-time monitoring of NPs in physiologically relevant milieu, many aspects are not addressed when screening DDS candidates. Recently, a Förster resonance energy transfer (FRET) approach was used to follow the disassembly of supramolecular structures in blood serum, ${ }^{14-17}$ and in animal models, ${ }^{18}$ by encapsulation or covalent attachment of a FRET fluorophore pair into one micelle. In our recent work, we employed PEG-Dendron amphiphiles that were functionalized with a spectral-shifting coumarin to track the stability of micelles in serum and cells. ${ }^{19}$ These micelles can selfreport their disassembly by the change of their fluorescent spectrum to the intrinsic emission of the labeling coumarin dyes. The change in assembly was detected in complex media using spectral imaging, enabling the NPs stability study in space and time. Nevertheless, the in vitro assays consist mainly of $2 \mathrm{D}$ systems, which despite being high throughput and rapid, do not provide complete information reflecting the in vivo conditions. ${ }^{20}$ In the last years, microfluidic 3D models have been extensively used to study cancer cells migration, ${ }^{22-25}$ vascularization and angiogenesis, $^{26-29}$ EB permeability, ${ }^{30-32}$ impact of 3D cell architecture, ${ }^{33-35}$ and tumor penetration. ${ }^{34,36-38}$ Recently, more complex blood vessel models have been designed to assess the differences in nanoparticles permeation in various conditions, ${ }^{13,30,31,39,40}$ demonstrating an impact of cancer cells on the endothelial permeability. However, in majority of them, only the intervals or the end time point of nanoparticles incubation are reported, lacking the important time-resolved information.

Herein, we address this challenge by combining a real-time spectral confocal imaging of polymeric, self-reporting micelles, which are perfused through tumor blood vessel model in a cancer-on-a-chip platform, to map their stability when encountering biological barriers. By evolving conventional 2D studies into more adequate 3D models, ${ }^{33,41,42}$ we aim to expand our previous work ${ }^{19}$ and provide additional screening before use of animal models. ${ }^{21,43-45}$

\section{RESULTS AND DISCUSSION}

The System: Amphiphilic PEG-Dendron Micelles and Microfluidic Cancer-on-a-Chip. Three amphiphilic PEGdendron hybrids, differing by their lipophilic end-groups in the hydrophobic block, were synthesized and characterized 

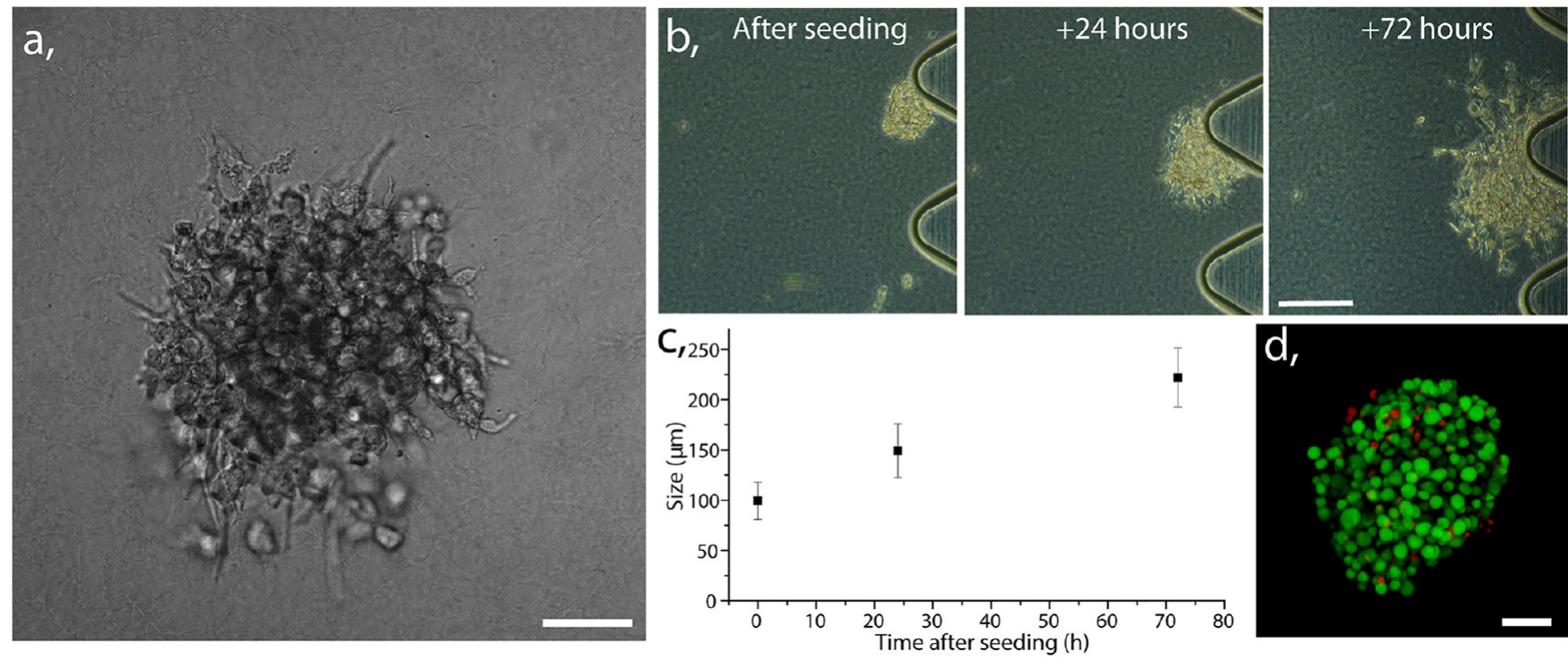

Figure 3. (a) Transmission image of collagen gel-embedded HeLa cells conformed into 3D spheroid. Scale bar $50 \mu \mathrm{m}$. (b) Transmission images demonstrating proliferation of a gel-embedded spheroid from the preparation up to 3 days in perfused culture. Scale bar $100 \mu \mathrm{m}$. (c) Graph demonstrating size progression of gel embedded spheroids after 1 and 3 days of perfused culture, calculated based on diameter measured in 10 different chips, average with standard error bars. (d) Confocal image of stained HeLa spheroid with calcein (live cells: green) and Propidium iodide (dead cells: red). Scale bar $50 \mu \mathrm{m}$.

following the previously reported methodology; ${ }^{19,46}$ the structures are shown in Figure 1a. The amphiphiles were labeled with a responsive dye, 7-diethylamino-3-carboxy coumarin, which forms an excimer when the hybrids selfassemble into micelles, resulting in a red-shift of the dye emission spectra, allowing to discriminate the assembled state from the disassembled state, as shown in Figure 1a. The responsive properties of these micelles, studied in the presence of serum proteins, HeLa cells, and at physiologically relevant temperature $\left(37{ }^{\circ} \mathrm{C}\right)$, have been previously demonstrated. ${ }^{19}$ Here we study the stability of these nanostructures in a canceron-a-chip platform and compare the results to the previously reported behavior in $2 \mathrm{D}$ cell culture.

In the cancer-on-a-chip model, we recapitulate the four important barriers that the micelles will have to overpass when injected into the body: (1) the flow of the blood vessel; (2) the endothelial barrier; (3) the ECM; and (4) the tumor spheroid (Figure 1b). To recreate this environment in a microfluidic platform, we used the 3D cell culture chip developed in Kamm's Lab and currently available commercially. ${ }^{47}$ It consists of three microfluidic channels: the central channel of $1.3 \times 0.25 \mathrm{~mm}^{2}(\mathrm{w}$ $\times \mathrm{h}$ ) and the two lateral media channels of $0.5 \times 0.25 \mathrm{~mm}^{2}(\mathrm{w} \times$ $\mathrm{h}$ ), as represented in Figures 1c and S1a. The middle channel is separated from the two lateral channels by rows of triangular microposts distant by $100 \mu \mathrm{m}$ from one another, as shown in Figure $1 \mathrm{c}$ and $\mathrm{f}$. These posts are designed to contain the unpolymerized gel (spheroids + collagen) in the central channel, by ensuring adequate surface tension, preventing gel leaks. In our model, one of the lateral channels represents the blood vessel and the middle channel recreates the ECM with embedded tumor spheroids as shown in the Figure 1d. Human umbilical vein endothelial cells (HUVECs) seeded in the lateral channel, lined it, and formed a lumen-like geometry, creating a vertical endothelial wall on the collagen gel scaffold (Figure 1e,f).

The created EB separates the inner lumen of the blood vessel model from the ECM channel, where we embedded preformed
HeLa spheroids into the Type 1 Collagen Gel (Figure 1f). The coculture of endothelial and cancer cells in the same systems adds complexity to the model; therefore, growth kinetics of HeLa cells and HUVECs were evaluated to determine the optimal medium for the healthy growth of both cell lines (see Supporting Figure S2). Overall, we recreated elements of tumor microenvironment in cancer-on-a-chip, where micelles stability can be evaluated during unidirectional perfusion through the blood vessel model channel. The parallel channel geometry of our platform enables continuous imaging of the nanocarrier interactions with the indicated barriers, as represented in Figure 1e. Once the tumor blood vessel model was recreated (continuously perfused with cell culture medium at $37{ }^{\circ} \mathrm{C}$ and $5 \% \mathrm{CO}_{2}$ ), the chip was placed in an optical confocal microscope, at $37{ }^{\circ} \mathrm{C}$, and reconnected to the perfusion system to track the assembly state of Hybrids 1-4 (see representation in Figure 1e and SI Supporting Videos).

Characterization of Reconstructed Barriers in Microfluidic Cancer-on-a-Chip Model. To validate our model, first we characterized the formation of an endothelial barrier created in the lateral channel. The magnified transmission image of the blood vessel channel and adjacent ECM channel with embedded HeLa spheroid (Figures 2a and S3) demonstrates the complete, prepared chip after 3 days of unidirectional medium perfusion. HUVECs are present in both the upper and lower plane of the blood vessel model, as shown in the transmission images of Figure $2 \mathrm{~b}$, and the formation of confluent endothelial monolayer lining the lumen was further validated by fixing and staining the cells, as shown in confocal image in Figure $2 c$ and $d$, where actin (red), nucleus (blue), and formation of tight junctions (green) can be observed. The endothelial cell to cell contact results in the expression of zonula occludens-1 (ZO-1) protein, which is essential to form these junctions in a healthy endothelial barrier. $^{48}$ Moreover, 3D reconstruction of confocal imaging demonstrates the presence of the EB between the microposts, on the gel scaffold (Figure 2d), physically separating the lumen of the vessel from the ECM channel, mimicking the in vivo barrier. 

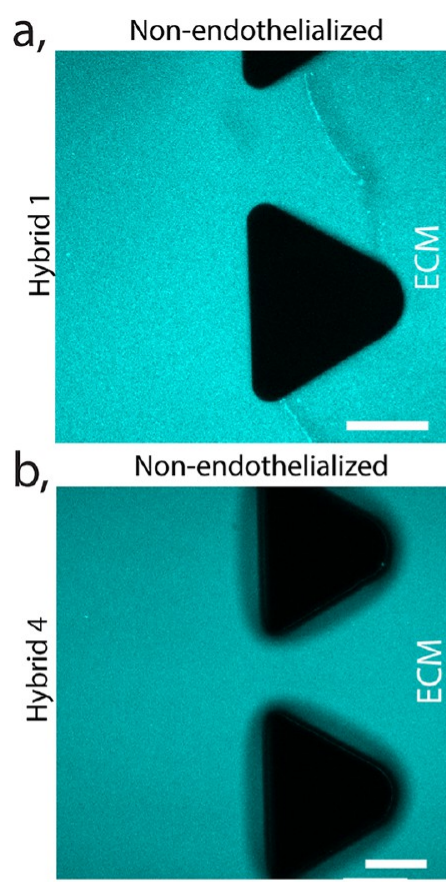

$C_{\text {I }}$

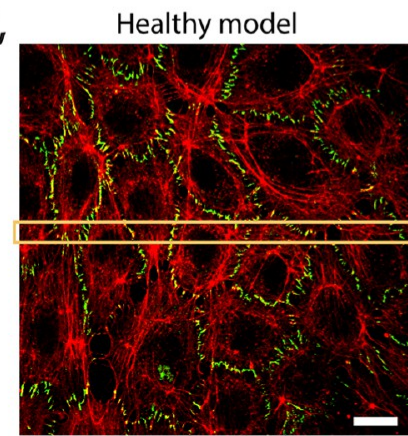

Healthy model

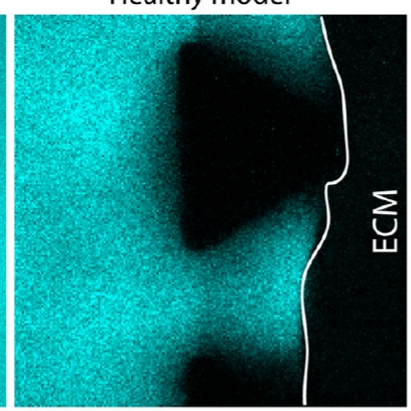

Healthy model

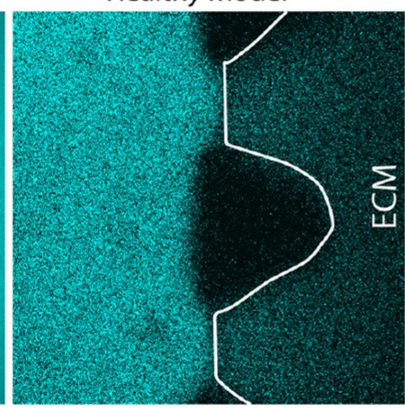

Cancer model

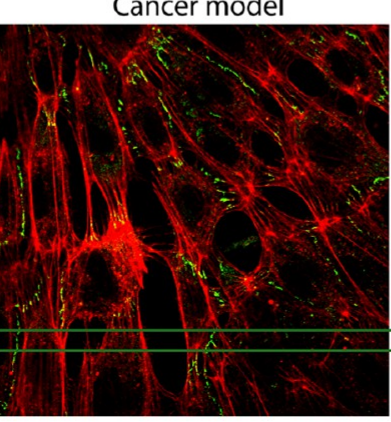

Cancer model

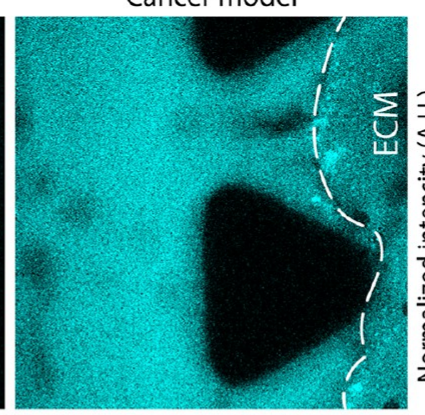

Cancer model

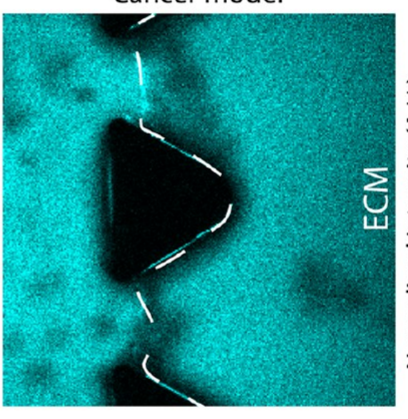

2. Non-endothelialized
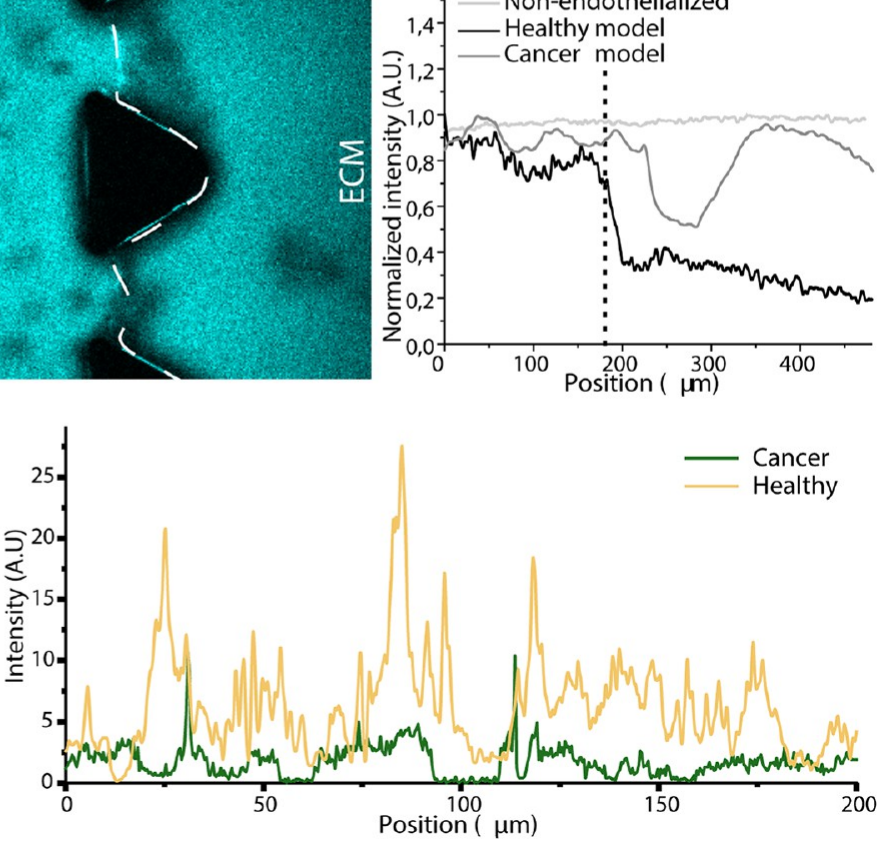

Figure 4. Extravasation of PEG-dendron hybrids in nonendothelialized, healthy and cancer models. (a) Confocal image of hybrid 1 extravasation in a (i) control chip (no EB), (ii) a healthy model, and (iii) cancer model. The images show emitted intensity between 446 and 700 nm, which include both the monomer and micelle signal. Scale bar $100 \mu \mathrm{m}$. Quantification of the normalized micelle fluorescence intensity measured in a rectangular area between two neighboring posts, indicating the penetration from the blood vessel model channel into the ECM part, plotted for the i-iii models. Vertical dotted line indicates the localization of the EB. (b) Confocal image of hybrid 4 (monomer) extravasation in the three corresponding models. Scale bar $100 \mu \mathrm{m}$. Quantification of the normalized monomer fluorescence intensity, measured in a rectangular area between two neighboring posts, indicating the hybrid 4 penetration from the blood vessel model channel into the ECM part, plotted for the i-iii models. Vertical dotted line indicates the localization of the EB. (c) Confocal image of HUVECs monolayer lining the healthy (left) and cancer (right) blood vessel channel model. Actin (red) and ZO-1 (green). Scale bar $20 \mu \mathrm{m}$. Quantification of the fluorescence intensity over the two marked rectangular areas indicated in the images (on the left). The spikes in the healthy model (yellow) originated from higher expression of ZO-1 in contrast to lower expression in the cancer model (green).

Additionally, HUVECs exhibited parallel alignment to the flow direction as a result of the created shear stress ${ }^{49,50}$ (Figure 2e). In contrast, cells cultured in static conditions showed random filament organization, as observed previously. ${ }^{51}$ Finally, we tested the structural integrity of the HUVECs barrier by measuring the retention of fluorescently labeled $10 \mathrm{kDa}$ Dextran, continuously perfused through the endothelialized channel. The fluorescence signal was detected in the lumen of the EB channel but not in the collagen gel (Figure 2f), indicating proper functionality of the endothelial barrier. However, a low gradual penetration of the Dextran into the ECM was observed after 30 min (Supporting Figure S4), similar to other reported studies. ${ }^{47,52-54}$ Altogether, these measurements indicate the formation of a functional EB with good structural integrity.

Finally, we characterized the ECM central channel with gel embedded HeLa spheroids. The transmission microscopy allowed us to observe the 3D spheroid conformation (Figure 3a) recapitulating aspects of geometry present in physiological conditions. This arrangement implies less available surface area per cell than in $2 \mathrm{D}$ cell culture models; it also alters cell proliferation rate and its overall functionality. ${ }^{20,34,55}$ The growth of prefomed spheroids, cocultured in the perfused chip together with HUVECs, is demonstrated in Figure $3 \mathrm{~b}$. The spheroids with size of $100 \pm 50 \mu \mathrm{m}$ were introduced into the chip, and after 3 days of culturing, they grew on average by $220 \%$ of their initial size, as shown in graph of Figure $3 \mathrm{c}$. HeLa viability was confirmed with a live/dead staining assay, revealing that the cells were viable throughout the spheroid after 3 days of culture (Figure $3 \mathrm{~d}$ ), indicating good nutrients and oxygen diffusion. Given the size of our spheroid after 3 days, we expected to observe high viability, as the phenomena of necrotic core 


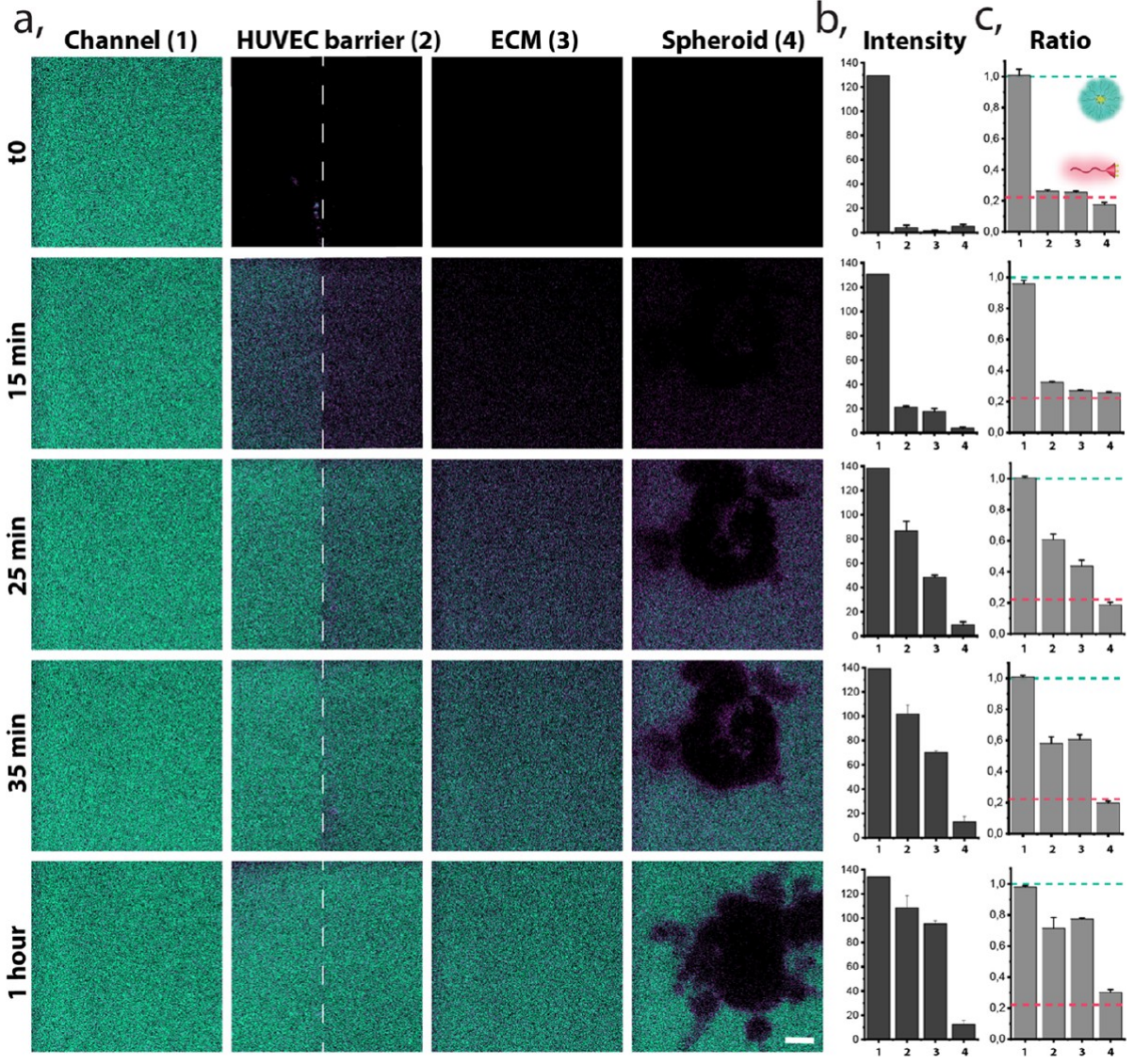

Figure 5. Space and time-resolved stability of hybrid 1. Representative images, selected from the 10 repetitions of experiments within hybrid 1 in the tumor blood vessel model. (a) Ratiometric confocal images of real-time monitored micelle (green) and monomer (magenta) at reconstructed barriers (1, blood vessel model channel; 2, HUVECs barrier; 3, ECM; 4, HeLa spheroid) during continuous perfusion of the hybrid 1, scale bar $15 \mu \mathrm{m}$. Relevant time points were selected for representative purposes. (b) Time-resolved intensity of fluorescence signal (A.U) originating from the sum of both: micelle and monomer channels at each barrier (1-4) of the presented images. (c) Normalized ratio of fluorescence signal between micellar and monomer form monitored in time at different barriers. Green dashed line indicates the ratio of fully formed micelles in equilibrium, and the magenta dashed line indicates the ratio of fully disassembled (monomer) form.

development is reported in the spheroids beyond $500 \mu \mathrm{m}$ diameter. ${ }^{56-59}$

Overall, we adapted a microfluidic platform to a $3 \mathrm{D}$ dynamic tumor microenvironment, including a perfusable blood vessel model, and cancer cells conformed into spheroid.

Increased Extravasation of Micelles Is Induced in Tumor Blood Vessel Model. Having established our tumor blood vessel-on-a-chip model, we investigated the ability of the micelles to penetrate the EB into the ECM channel. Previous studies using microfluidic models reported enhanced permeability of endothelial cells when exposed to specific molecules, such as TNF- $\alpha^{39}$ or when cocultured with cancer cells, ${ }^{31}$ leading to the formation of "leaky vessels", representing one of the features of the EPR effect. However, most of these microfluidics models were used for studying only the selected or final time point of nanoparticles penetration. Herein, taking advantage of the characteristics of the chip, we could continuously monitor the perfusion of our micelles in three different experimental conditions: (i) no HUVECs (nonendothelialized lateral channel) as negative control; (ii) HUVECs barrier (healthy endothelialized blood vessel model), and (iii) HUVECs barrier with HeLa spheroids embedded in the ECM (tumor blood vessel model).

First, we tested the micelle ability to cross the EB, perfusing the hybrid $\mathbf{1}$ into the three models (i-iii) and quantifying the fluorescence intensity, in the blood vessel model and ECM part, as plotted for the i-iii in Figure 4a. A constant amount of the fluorescence was immediately detected in the collagen gel in case of lack of the EB (i), which demonstrates that our NPs freely diffuse through the ECM. ${ }^{5}$ On the contrary, the majority (>90\%) of hybrid 1 was retained in the healthy blood vessel model channel (ii) for more than $30 \mathrm{~min}$ of continuous perfusion. Meanwhile, a gradual diffusion of the hybrid into the ECM was observed in the tumor blood vessel model (iii). These results indicate that the HUVECs monolayer in the healthy model fulfills the barrier function and limits the hybrid penetration into the ECM. However, this function is affected by presence of HeLa spheroids in the gel. Our observations resemble experiments performed by Tang and co-workers where coculture of cancer endothelial cells with breast cancer cells increased the permeation of nanoparticles through the EB. ${ }^{31}$

Interestingly, we hypothesized that the $\sim 10 \%$ of the hybrid detected in the ECM of the healthy model (ii) could originate from the infiltration of the monomer (disassembled form $\sim 6-7$ $\mathrm{kDa}$, assembled micelle $\sim 20 \mathrm{~nm}$ ), small enough to pass through the healthy EB. ${ }^{47,60}$ To investigate this further, we perfused hydrophilic hybrid 4, which has four hydroxyl end-groups (does not self-assemble into micelles), ${ }^{19}$ into the same three models (i-iii) as hybrid 1. As expected, Figure $4 \mathrm{~b}$ shows that the monomer penetrated the ECM instantly in the control (i) and 
the cancer (iii) models, but some infiltration into the ECM was also observed in the healthy (ii) one. Specifically, HUVECs barrier partially retained the monomer for 25 min of continuous perfusion, limiting its concentration in the gel to less than $40 \%$ of its intensity in the perfused channel. It is worth noting that after 5 min of perfusion, the hybrid 4 could already be detected in the gel of the healthy blood vessel model (Supporting Figure S6). However, the penetration into the ECM was far more significant and immediate in the HeLa cells cocultured chip. Overall, we hypothesize that the monomer form can gradually cross into the ECM region of the healthy blood vessel model due to its small size, permitting the paracellular transport.

We visually determined the morphological effect of coculturing cancer cells on the structural integrity of the endothelial monolayer to understand if a loss of EB integrity was the reason for the increased micelles permeation into the ECM in the cancer model. Tight and adherent junctions are the crucial structural elements formed between endothelial cells, regulating paracellular diffusion and restricting the permeation of molecules bigger than $\sim 2 \mathrm{~nm} .{ }^{61}$ To confirm that the enhanced permeability of the EB in our model was induced by the presence of HeLa spheroids, we prepared the chips as mentioned previously, and after 3 days of medium perfusion, the cells were fixed, ZO-1 protein stained, and quantified as shown in Figure 4c and Supporting Figure S7. ZO-1 was clearly and uniformly expressed between HUVECs of the healthy model; however, the expression was reduced in the HeLa spheroids cocultured model. It indicated that cancer cells impacted the HUVEC cell-cell interaction and the tight junction formation, therefore explaining the enhanced permeability in our cancer models. Similar findings were reported by Kaji et al., ${ }^{62}$ where HUVEC and HeLa coculture affect the endothelial cells growth through the direct cell-cell contact as well as transmission of information via culture medium (paracrine communication). In that study, the cytokines excreted by HeLa repulsed HUVECs and released reactive oxygen species, which led to malfunction and death of HUVECs, resulting in leakiness of the EB. However, it is worth noting that in our study there was a certain heterogeneity in the permeation of micelles out of the tumor blood vessel model. We observed that small variations in the number of spheroids (or their distribution) affect the EB retention capacity, potentially resulting in a variable concentration of signaling molecules (see Supporting Discussion). These observations may be reflecting one of the key features of the EPR effect, of which the heterogeneity has been extensively discussed recently, and attributed to the stage of the diseases. ${ }^{63}$

Time- and Space-Resolved Micelle Stability Revealed in 3D Tumor Microenvironment Model. The aim of our work was to study the stability of our micellar systems when introduced into the microfluidic 3D model. Previously the micelles and monomer were detected in the presence of serum proteins and their internalization pathway identified thanks to their self-reporting capabilities compatible with confocal fluorescence microscopy. ${ }^{19}$ Herein, we hypothesized that the added complexity and dynamicity of the blood vessel model may induce premature disassembly due to multiple interactions. To evaluate these critical interactions, we continuously perfused micelles of hybrid 1 (the most stable system), in full culture medium at $15 \mu \mathrm{L} / \mathrm{min}$ and $37^{\circ} \mathrm{C}$ into the blood vessel model channel. During perfusion, we continuously monitored the micelles' stability in key regions: the blood vessel model channel, the endothelial barrier, the ECM, and the HeLa spheroids, providing real-time stability information as shown in Figure 5 and the Supporting Videos.

In the first minutes of perfusion, fluorescence was detectable only in the blood vessel model channel (Figures 5a,b) and indicated the presence of assembled micelles (Figure 5c). Hybrid penetration into the depth of the ECM was observed over time. After $15 \mathrm{~min}$ of continuous flow, the hybrid started to reach the EB, and we could observe that the disassembled form prevailed in passing through the wall and entering the ECM by detecting the monomer emission (magenta). After $25 \mathrm{~min}$, we observed the assembled form traversing the EB, while the deep penetration into ECM was still achieved mostly by the disassembled polymers. This observation could be attributed to two factors: (i) the micelles progressively overcame the endothelial barrier or (ii) the monomer form, which entered the ECM through the EB previously, accumulated, and reached the critical micelle concentration (CMC), reassembling into micelles. Figure $5 \mathrm{~b}$ demonstrates that both, micelle and monomer, coexist at the EB and in the ECM with the mean ratio 0.6 and 0.4 , respectively. The assembled structures were detected in the surroundings of the spheroids after more than half an hour of the continuous perfusion. Surprisingly, after $1 \mathrm{~h}$, we observed only weak penetration of the hybrid 1 into the depth of the HeLa spheroids, similarly to $2 \mathrm{~h}$ of constant perfusion (Supplementary Figures S8 and S9). In our previous 2D cell internalization studies, the hybrids were detected inside the cell already after $10 \mathrm{~min}$; meanwhile, in the current work, only a small fraction of the disassembled form was detected in the outer layer of the spheroid.

Interestingly, we observed a stabilization of monomer/micelle equilibrium in the monitored regions after $1 \mathrm{~h}$ from the beginning of the perfusion, except for the spheroid area. Hybrid 1 , detected as a monomer in contact with HeLa cells, was a contrasting observation comparing to our previously reported internalization behavior in 2D cell cultures, where the assembled hybrid 1 was taken up by HeLa cell via endocytosis, and its disassembly progressed in time. ${ }^{19}$ This discrepancy can be attributed to the spheroid form of the HeLa, promoting different endocytosis process, favored in the new $3 \mathrm{D}$ cells confluency and conformation. Other works, highlighting the importance of going beyond 2D cell culture models, investigated the penetration of nanosystems into tumor spheroids as a function of nanocarrier size, shape, charge, and functionalization. ${ }^{36,64,65}$ Likewise, the penetration of cross-linked and non-cross-linked micelles has been compared, showing an improved result for the cross-linked (more stable) ones. ${ }^{66-68}$ Therefore, we hypothesized that the lower spheroid penetration in our model can be caused by a premature disassembly in the periphery of the spheroid leading to a different outcome than reported in the $2 \mathrm{D}$ static monoculture. It underlines the importance of model selection in rational evaluation and optimization of supramolecular systems for drug delivery.

Stability of Hybrids Dictates Their Infiltration/Extravasation. Finally, we investigated the interplay between molecular structure, micellar stability, and their ability to extravasate. Therefore, we compared the stability of three hybrids, with decreasing length of the hydrophobic end-groups, from hybrid 1 to hybrid 3 . In our previous $2 \mathrm{D}$ studies, we demonstrated that the hybrid $\mathbf{1}$ was stable in the presence of serum proteins and upon dilution; meanwhile, the stabilities of hybrid $\mathbf{2}$ and $\mathbf{3}$ were similar when diluted with serum; however, their disassembly kinetics were significantly different. While 

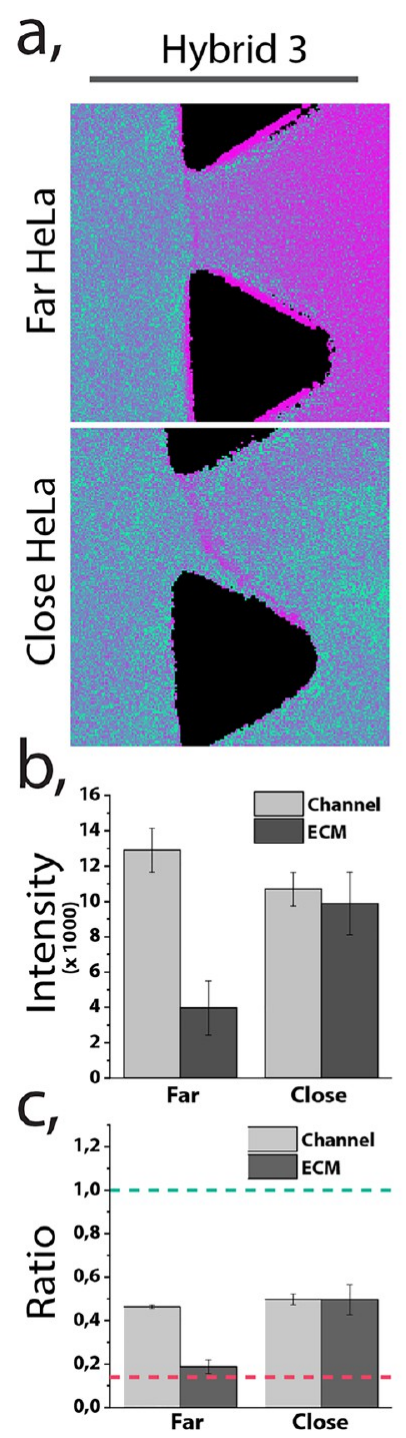

Hybrid 2
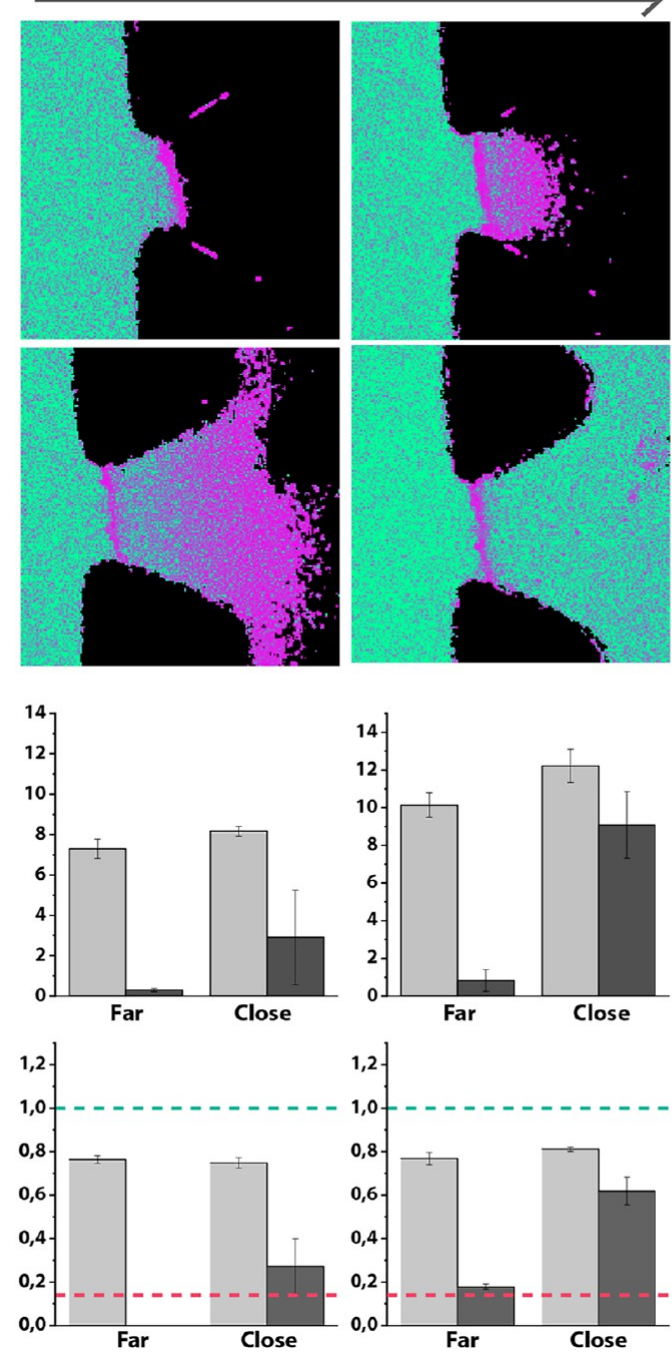

time

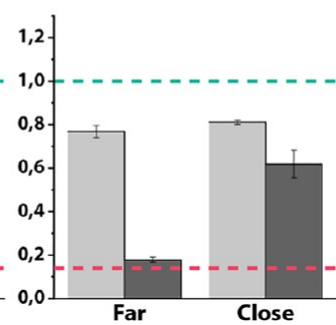

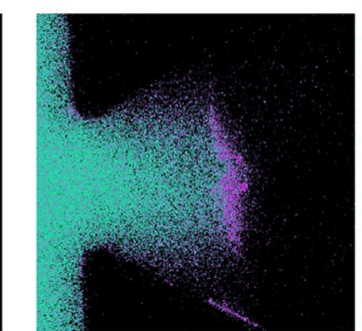

Hybrid 1
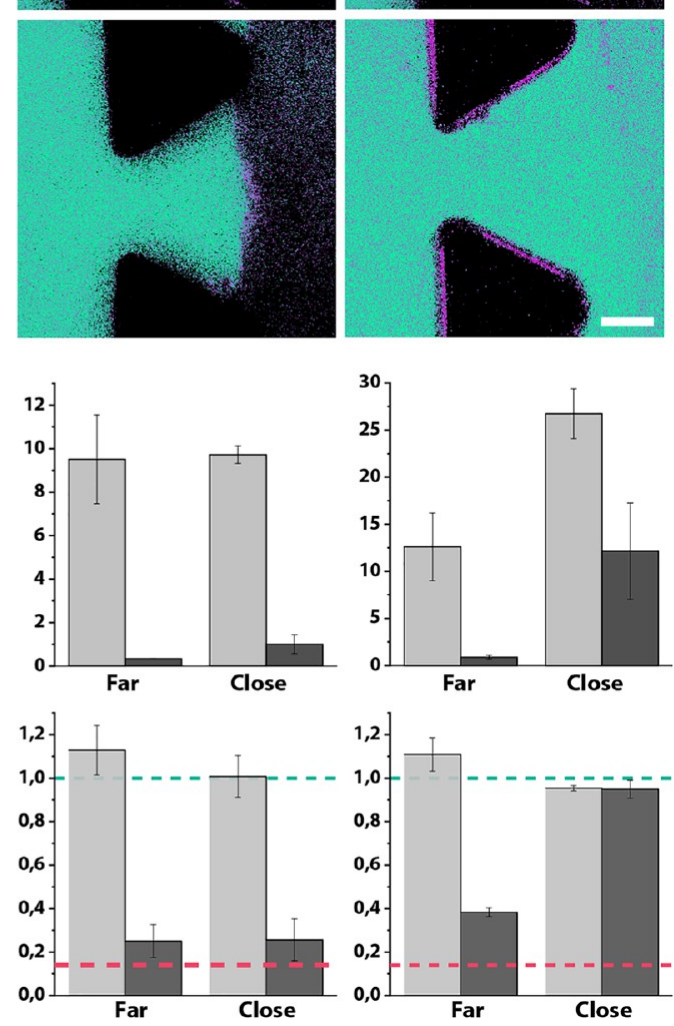

Figure 6. Stability of hybrids $1-3$, perfused through a tumor blood vessel models with different EB distance to the HeLa spheroid (close: < $400 \mu$ m, far: $>1 \mathrm{~mm}$ ). (a) Ratiometric confocal images of the different hybrids perfused through the chip in two regions. Hybrids $\mathbf{1}$ and $\mathbf{2}$ are shown at two different time points: less than $15 \mathrm{~min}$ and after $30 \mathrm{~min}$ of continuous perfusion; hybrid 3 demonstrated at one time point due to its rapid penetration through the EB. Scale bar $75 \mu \mathrm{m}$. (b) Summed up fluorescence intensity originating from the monomer and the micelle channels. Intensity was measured in the blood vessel model channel and in the ECM region. (c) Normalized ratio of fluorescence signal between micellar and monomer form for each hybrid and in each region. Green dashed line indicates the ratio of fully formed micelles in equilibrium, and the magenta dashed line indicates the ratio of fully disassembled (monomer) form.

hybrid 3 disassembled rapidly upon dilution, hybrid 2 needed hours to reach equilibrium.

To understand how these differences in thermodynamics and kinetic stability are reflected in a more complex model, we perfused each hybrid solution (in full culture media) through the blood vessel model channel during at least $30 \mathrm{~min}$. In Figure 6, we show representative images, demonstrating observations for the sets of hybrid-cancer model experiments, where an individual chip was perfused only with one hybrid. We chose two different areas of each chip-hybrid set, taking the distance of the HeLa spheroid to the HUVECs barrier as the selection criterium, to illustrate the different behavior we observed. HeLa spheroids less than $400 \mu \mathrm{m}$ from the EB were considered as "close" to HeLa and EB regions at a radius of at least $1 \mathrm{~mm}$ were considered as regions "far" from HeLa, as reported in Figure 6 and Supporting Figure S10. We observed extravasation of all hybrids 1-3 through the EB when HeLa spheroids were located close to HUVECs (Figure 6b). However, in the distant regions (far HeLa), only hybrid 3 (which has the least hydrophobic dendron) was able to significantly extravasate to the ECM. Thus, we concluded that the endothelial barrier "leakiness" can be heterogeneous, depending on the amount, distance, and distribution of tumor spheroids in the ECM.

Monitoring stability of these three hybrids (Figure 6a and c, and Supporting Videos) was based on the ratios between the emitted intensity of the coumarin dye at 480 and $550 \mathrm{~nm}$ (the disassembled and assembled state respectively). Hybrid $\mathbf{1}$ appeared as a micelle in the blood vessel model channel, with mean ratio of fluorescence signal between the both forms equal to 1 (indicative of the micelle), while slight disassembly of hybrid $\mathbf{2}$ and significant disassembly of hybrid 3 were observed, with mean ratios of 0.8 and 0.5 , respectively. Our previous study showed that hybrids 2 and 3 were slightly unstable in the presence of serum proteins, but their degree of disassembly based on the fluorescent ratio was equal. Therefore, we hypothesized the enhanced disassembly of hybrid 3 is not only 
due to interactions with serum proteins but also caused by the perfusion. This result indicates that the flow-induced shear stress can drastically affect the stability of supramolecular nanocarriers.

Furthermore, we monitored the stability of each hybrid at the previously defined barriers and observed significant differences among them. Interestingly, in regions far from spheroids, only the monomeric form of hybrid 3 was able to efficiently extravasate. This phenomenon could occur due to the increased disassembly of the micelle in contact with the HUVEC barrier, which allowed the monomer to (i) paracellularly extravasated due to its small size or (ii) transcellularly cross the EB. In contrast to that, hybrid 3 crossed the EB in the semiassembled state in the regions close to HeLa (as it appeared in the blood vessel channel), probably due to the disappearance of the tight junctions in the HUVEC barrier. On the other hand, the extravasation of hybrid 2 in regions close to HeLa had a timedependent response; first only monomer crossed the EB and the micelles were detected in the ECM after more than $30 \mathrm{~min}$. Finally, we observed that hybrid $\mathbf{1}$ behaves similarly to the hybrid 2, where monomer molecules extravasated first, followed by the later penetration of the assembled micelles. Interestingly, while hybrids 2 and 3 rapidly accumulated in the endothelial barrier only as a monomer, hybrid 1 monomeric form accumulated at the EB only in areas far away from the HeLa cells. Previously, in 2D cell culture, hybrids 3 and $\mathbf{2}$ internalized as monomer, and the hybrid $\mathbf{1}$ internalized as a micelle and disassembled over time. ${ }^{19}$ Overall, we could correlate the interplay between stability of the micelles and their performance in a $3 \mathrm{D}$ model as well as their ability to extravasate and reach the cancer cell regions.

\section{CONCLUSIONS}

In the present work, we combined spectral confocal imaging and a microfluidic cancer-on-a-chip model as a new approach to study the stability of supramolecular nanocarriers. The fluorescence properties of our micelles allowed tracking of their assembly state across the changing conditions of the reconstructed elements of tumor microenvironment.

The results show the formation of functional endothelial barrier in a lumen of the blood vessel model and appearance of leaky vasculature in the coculture with cancer cells. The permeable endothelial wall displays heterogeneity, dependent on the number and distance of HeLa spheroids, resembling to some extent the in vivo pathologies of many tumors.

We obtained a precise and direct information about the performance and stability of the micelles in each of the barriers, thanks to the time and space-resolved imaging. We reported the ability of the most stable hybrids $\mathbf{1}$ and $\mathbf{2}$ to extravasate from the blood vessel model as assembled micelles, while the shear stress and interactions with the EB induced disassembly of the hybrid 3 . Therefore, we believe these two micelles are the best candidates to be used as DDS in vivo. However, we observed the loss of stability of hybrid $\mathbf{1}$ in proximity of the spheroids, as well as a poor penetration into its depth, which indicated a need for improvement to achieve good in vivo efficacy.

Our approach, combining spectrally responsive supramolecular structures with a cancer-on-a-chip platform, has the capacity to provide new knowledge about nanoparticles performance, stability, and accumulation in tumor, which is essential to bridge the gap between in vitro and in vivo testing of new drug delivery systems.

\section{MATERIALS AND METHODS}

Microfluidic Device and Setup. Microfluidic 3D culture chip DAX-1 (AIM Biotech) was used as a platform to reconstruct tumor microenvironment (blood vessel model channel and ECM with embedded spheroids). LUC-1 connectors (AIM Biotech) were used to connect the chip inlets with luer connector ended PTFE tubing to facilitate the continuous perfusion. The other end of the tubbing was connected to a syringe placed in a double syringe pump (Nexus Fusion 200) and filled with HUVEC (EndoGRO, Millipore) basal medium, used to constantly perfuse the chip for $48-72 \mathrm{~h}$.

Cells and Reagents. Human umbilical vein endothelial cells (Promocell) were used to recreate blood vessel lining in the blood vessel model channel, and HeLa cells were used in to create tumor spheroids. HUVECs were cultured in EndoGRO Basal medium (Millipore) supplemented with SCME001 kit (EndoGRO-LS Supplement $0.2 \%$, rh EGF $5 \mathrm{ng} / \mathrm{mL}$, ascorbic acid $50 \mu \mathrm{g} / \mathrm{mL}$, L-glutamine $10 \mathrm{mM}$, hydrocortisone hemisuccinate $1 \mu \mathrm{g} / \mathrm{mL}$, heparin sulfate $0.75 \mathrm{U} / \mathrm{mL}$, FBS $2 \%$ ), and penicillin/streptomycin $1 \%$ (Biowest). HeLa cells were cultured in Dulbecco's modified Eagle medium (DMEM, as received with L-glutamine, $4.5 \mathrm{~g} / \mathrm{L}$ D-glucose and pyruvate, Gibco) supplemented with FBS 5\% (Gibco) and penicillin/streptomycin 1\% (Biowest). HUVECs were cultured in $75 \mathrm{~cm}^{2}$ flasks and HeLa in $25 \mathrm{~cm}^{2}$ flasks at $37^{\circ} \mathrm{C}$ and $5 \% \mathrm{CO}_{2}$. Cells were harvested using trypsin-EDTA $(0.25 \%$, Gibco) when they reached $70-80 \%$ confluence.

Cell Culture in the Microfluidic Device. Collagen gel at concentration of $2.5 \mathrm{mg} / \mathrm{mL}$ was prepared, introduced, and polymerized according to the general protocol v5.3 (AIM Biotech). In brief, Rat tail collagen Type I (Corning Life Science) was mixed on ice with 10x PBS (Sigma-Aldrich) and Phenol Red (Sigma-Aldrich), and the $\mathrm{pH}$ of the mixture was adjusted to 7.4 using $0.5 \mathrm{M} \mathrm{NaOH}(\mathrm{NaOH}$ in pellets PanReac dissolved in MiliQ water); final volume was adjusted with MiliQ water (for healthy model) or with suspension of HeLa clusters (for cancer model).

For preparation of the cancer model, microfluidic chip HeLa cells were seeded into a 96-well ultralow attachment plate (Corning) at $0.5-1.5 \mathrm{k}$ cells/well and cultured for $72 \pm 24 \mathrm{~h}$. Formed cell spheroids were harvested, centrifuged, and resuspended in previously prepared collagen gel, resulting in few clusters (of $100 \pm 50 \mu \mathrm{m}$ ) per $10 \mu \mathrm{L}$ of the gel at the concentration of $2.5 \mathrm{mg} / \mathrm{mL}$. Prepared collagen was inserted into the central channel of 3D culture chip and allowed to polymerize during $30 \mathrm{~min}$ at $37{ }^{\circ} \mathrm{C}$ and $5 \% \mathrm{CO}_{2}$. After gel polymerization, one of the lateral channels was prepared for HUVECs culture by coating the channel with $50 \mu \mathrm{g} / \mathrm{mL}$ fibronectin (FN) from bovine plasma (Sigma-Aldrich) for $2 \mathrm{~h}$ at $37{ }^{\circ} \mathrm{C}$ and $5 \% \mathrm{CO}_{2}$. The remaining lateral channel was filled in with DMEM (HeLa culture medium) and closed using luer caps.

After the incubation time, the FN was washed away using $1 \mathrm{x}$ PBS (Gibco) and EndoGRO HUVEC medium. HUVECs were seeded in the prepared lateral channel at a density of $2.5-3.5 \mathrm{M}$ cells $/ \mathrm{mL}$. The $3 \mathrm{D}$ culture chip was flipped upside down to allow cell adhesion to the upper plane for $1.5-2.5 \mathrm{~h}$ at $37^{\circ} \mathrm{C}$ and $5 \%$ $\mathrm{CO}_{2}$. A second batch of HUVECs cultured in another flasks was harvested and introduced to the same lateral channel at the same concentration as previously. The cells were then incubated for minimum $2 \mathrm{~h}$ at $37{ }^{\circ} \mathrm{C}$ and $5 \% \mathrm{CO}_{2}$ in the upright position to allow their attachment to the lower plane. Next, the chip was perfused with EndoGRO HUVEC medium at a flow rate 3-5 $\mu \mathrm{L} / \mathrm{min}$ for $48-72 \mathrm{~h}$ (as described above) until HUVECs reached confluency.

Hybrids Perfusion Setup. Hybrids 1-4 were prepared at a concentration of $480 \mu \mathrm{M}$ in filtered PBS, sonicated for $5 \mathrm{~min}$, and let to equilibrate for at least $10 \mathrm{~min}$. Prior to hybrid flowing into the chip, they were mixed with full EndroGRO HUVEC medium resulting in final concentration of $160 \mu \mathrm{M}$. 
The microfluidic chip was placed into the on-stage incubator of a Zeiss LSM 800 confocal microscope at a temperature of 37 ${ }^{\circ} \mathrm{C}$ and $5 \% \mathrm{CO}_{2}$, and connected to peristaltic pump (Ismatec, Reglo Digital, ISM597) with a silicone tubing (Tygon, Kinesis) to perfuse hybrids during real-time imaging at $15 \mu \mathrm{L} / \mathrm{min}$. Hybrids were excited using a $405 \mathrm{~nm}$ laser, and emission spectra were collected using two different PMT detectors to detect both monomer and micelle separately and simultaneously. The windows of detection were set as follows: (i) monomer 446$500 \mathrm{~nm}$ and (ii) micelle 500-700 nm. Ratiometric images were obtained from dividing the micelle image by the monomer image after a mask was applied to each image where noise was removed.

To calculate the amount of hybrid able to extravasate, we first summed up the signal of both windows. Next, we calculated the mean intensity signal of the vessel channel and used this value as the maximum concentration. Next the mean intensity signal of the gel channel was calculated and divided by the maximum signal concentration.

Dextran Perfusion. The $10 \mathrm{kDa}$ Dextran labeled with AlexaFluor568 (Thermo Fisher Scientific) was diluted in HUVEC (EndoGRO) medium at a final concentration of 1 $\mu \mathrm{g} / \mathrm{mL}$. The solution was perfused into the blood vessel model channel at a flow rate of $5 \mu \mathrm{L} / \mathrm{min}$ using the syringe pump. The perfusion of dextran was monitored using Nikon Eclipse Ti2 epifluorescent microscope. The chip was placed in the on-stage incubator (OKOlab) at a temperature of $37^{\circ} \mathrm{C}$ and $5 \% \mathrm{CO}_{2}$, the perfused fluorophore was excited at $525 \mathrm{~nm}$, and the emission was collected at $650 \mathrm{~nm}$.

HeLa Spheroid Viability Assay. The viability of HeLa cells within the spheroids was evaluated using Calcein (Fluka, SigmaAldrich) and propidium iodide (Sigma-Aldrich) to stain live and dead cells, respectively. First, cells were incubated with $10 \mu \mathrm{M}$ Calcein solution for $20 \mathrm{~min}$ at $37^{\circ} \mathrm{C}$ and $5 \% \mathrm{CO}_{2}$. Next, the cells were incubated with $10 \mu \mathrm{g} / \mathrm{mL}$ propidium iodide solution for 5 min at $37{ }^{\circ} \mathrm{C}$ and $5 \% \mathrm{CO}_{2}$ and then washed with $1 \times$ PBS (SigmaAldrich). The imaging was performed using a Zeiss LSM 800 confocal microscope. The Calcein and propidium iodide stained spheroids were excited at laser wavelength of 488 and $561 \mathrm{~nm}$, respectively, and detection windows set at 400-600 nm for Calcein and $600-700 \mathrm{~nm}$ for propidium iodide. The 3D image was reconstructed (ZEN, confocal microscope software) from slices acquired in a Z-stack mode with a plane interval of $1,5 \mu \mathrm{m}$.

Immunostaining, Labeling, and Confocal Microscopy (Confocal Imaging Labeling). Cells in the microfluidic chip were washed with 1x PBS (Gibco) and fixed with 4 wt \% solution of paraformaldehyde (PFA, Sigma-Aldrich) in 1x PBS. After 10 $\mathrm{min}$, and the fixative was washed away with $1 \mathrm{x}$ PBS, cells were permeabilized for $10 \mathrm{~min}$ with $0.1 \%$ solution of Triton X-100 (Sigma-Aldrich) in $1 \mathrm{x}$ PBS and exposed for $1 \mathrm{~h}$ to a $3 \%$ bovine serum albumin (BSA, Sigma-Aldrich) blocking solution in $1 \mathrm{x}$ PBS.

Next, the HUVECs' tight junctions were stained using $5 \mu \mathrm{g} /$ mL ZO-1 (Zonula Occludens-1) monoclonal antibody conjugated with Alexa Fluor 488 (Thermo Fisher Scientific) solution in previously prepared $3 \% \mathrm{BSA}$ during $\mathrm{O} / \mathrm{N}$ incubation at $4{ }^{\circ} \mathrm{C}$. In the next step, the cells were washed with $3 \%$ BSA solution and incubated with 1x Phalloidin-iFluor594 (Abcam, stock $1000 \mathrm{x}$ ) solution (in 1\% BSA) for $30 \mathrm{~min}$ at RT to stain actin filaments. The cell nuclei were stained after washing the cells with $1 \mathrm{x}$ PBS, using Hoechst 33258 stain at concentration 5 $\mu \mathrm{g} / \mathrm{mL}$. After $10 \mathrm{~min}$ of incubation at RT, the cells were washed with $1 \mathrm{x}$ PBS and imaged at RT using a Zeiss LSM 800 confocal microscope. Nuclei, tight junctions, and actin were excited using a $405 \mathrm{~nm}, 488 \mathrm{~nm}$, and $561 \mathrm{~nm}$ laser, respectively. The 3D images were acquired scanning the sample in a Z-stack mode, with an acquisition plane each 1 to $10 \mu \mathrm{m}$ and later reconstructed into $3 \mathrm{D}$ image using the $\mathrm{ZEN}$ (confocal microscope) software.

To calculate the orientation of actin filaments in static versus dynamic conditions, two independent chips were prepared as explained; however, one of the chips was incubated in static conditions, with medium change every $24 \mathrm{~h}$; meanwhile, the other was continuously perfused with cell medium. After $72 \mathrm{~h}$, the cells were fixed and actin stained, and confocal images of actin were acquired using Zeiss LSM 800. The images were analyzed using the OrientationJ plugin of ImageJ to obtain the distribution of the orientation's graphs.

\section{ASSOCIATED CONTENT}

\section{SI Supporting Information}

Supporting Figures and Discussion (PDF file) The Supporting Information is available free of charge at https://pubs.acs.org/ doi/10.1021/acsabm.0c01209.

Microfluidic chip used to recreate cancer-on-a-chip model, growth kinetics of HUVECs and HeLa, HUVECs monolayer formation, snapshots of continuous perfusion of Dextran through blood vessel model channel, epifluorescent microscopy 3D reconstructed image of continuously perfused Dextran through endothelialized blood vessel model channel, extravasation of hybrid $\mathbf{4}$ in healthy model, confocal images of $\mathrm{ZO}-1$ expression in HUVECs monolayer, space resolved stability of hybrid, penetration and stability of hybrid $\mathbf{1}$ in HeLa spheroids, transmission images of cancer model chip and corresponding ratiometric image, supporting discussion (PDF) Perfusion of hybrid $\mathbf{1}$ into healthy blood vessel model channel (AVI)

Continuation of perfusion of hybrid $\mathbf{1}$ into healthy blood vessel model channel (AVI)

Perfusion of hybrid $\mathbf{1}$ into tumor blood vessel model channel (AVI)

Ratiometric video of perfusion of hybrid $\mathbf{1}$ into tumor blood vessel model channel (AVI)

Perfusion of hybrid $\mathbf{1}$ into non-endothelialized lateral channel (AVI)

\section{AUTHOR INFORMATION}

\section{Corresponding Authors}

Lorenzo Albertazzi - Institute for Bioengineering of Catalonia, The Barcelona Institute of Science and Technology (BIST), 08024 Barcelona, Spain; Department of Biomedical Engineering, Institute for Complex Molecular Systems (ICMS), Eindhoven University of Technology, 5612AZ Eindhoven, The Netherlands; ○ orcid.org/0000-0002-6837-0812;

Email: 1.albertazzi@tue.nl

Roey J. Amir - Department of Organic Chemistry, School of Chemistry, Faculty of Exact Sciences, Tel-Aviv University, TelAviv 6997801, Israel; Tel Aviv University Center for Nanoscience and Nanotechnology, BLAVATNIK CENTER for Drug Discovery, and The ADAMA Center for Novel Delivery Systems in Crop Protection, Tel-Aviv University, TelAviv 6997801, Israel; (1) orcid.org/0000-0002-8502-3302; Email: amirroey@tauex.tau.ac.il 


\section{Authors}

Natalia Feiner-Gracia - Institute for Bioengineering of Catalonia, The Barcelona Institute of Science and Technology (BIST), 08024 Barcelona, Spain; Department of Biomedical Engineering, Institute for Complex Molecular Systems (ICMS), Eindhoven University of Technology, 5612AZ Eindhoven, The Netherlands

Adrianna Glinkowska Mares - Institute for Bioengineering of Catalonia, The Barcelona Institute of Science and Technology (BIST), 08024 Barcelona, Spain; Department of Biomedical Engineering, Institute for Complex Molecular Systems (ICMS), Eindhoven University of Technology, 5612AZ Eindhoven, The Netherlands

Marina Buzhor - Department of Organic Chemistry, School of Chemistry, Faculty of Exact Sciences, Tel-Aviv University, TelAviv 6997801, Israel; Tel Aviv University Center for Nanoscience and Nanotechnology, Tel-Aviv University, TelAviv 6997801, Israel

Romen Rodriguez-Trujillo - Institute for Bioengineering of Catalonia, The Barcelona Institute of Science and Technology (BIST), 08024 Barcelona, Spain; Department of Electronic and Biomedical Engineering, Faculty of Physics, University of Barcelona, 08028 Barcelona, Spain

Josep Samitier Marti - Institute for Bioengineering of Catalonia, The Barcelona Institute of Science and Technology (BIST), 08024 Barcelona, Spain; Department of Electronic and Biomedical Engineering, Faculty of Physics, University of Barcelona, 08028 Barcelona, Spain; Networking Biomedical Research Center in Bioengineering, Biomaterials and Nanomedicine (CIBER-BBN), 28029 Madrid, Spain

Silvia Pujals - Institute for Bioengineering of Catalonia, The Barcelona Institute of Science and Technology (BIST), 08024 Barcelona, Spain; Department of Electronic and Biomedical Engineering, Faculty of Physics, University of Barcelona, 08028 Barcelona, Spain

Complete contact information is available at:

https://pubs.acs.org/10.1021/acsabm.0c01209

\section{Author Contributions}

${ }^{\circ}$ N.F.-G. and A.G.M. contributed equally. N.F.-G. and A.G.M. executed entire experimental part. M.B. synthesized the hybrids. N.F.-G., A.G.M., S.P., L.A., R.J.A., and J.S.M. conceived and designed the project. All authors contributed in the interpretation of results and experimental design. N.F.-G. and A.G.M. wrote the manuscript, and all authors revised the final version.

\section{Notes}

The authors declare no competing financial interest.

\section{ACKNOWLEDGMENTS}

N.F.-G. and A.G.M. thank D. Izquierdo for the experimental support and scientific discussions. The authors thank the Biomaterials for Regenerative Therapies group in IBEC for sharing their equipment to execute the experiments and IBEC's Microscopy Characterization Facilities equipped with Confocal Microscope. L.A. and S.P. thank the Spanish Ministry of Science and Innovation, through the Project PID2019-109450RB-I00/ AEI/10.13039/501100011033, by the Generalitat de Catalunya through the CERCA program and 2017 SGR 01536. The authors also acknowledge the EuroNanoMed II platform through the project NANOVAX (PCIN-2016-025) and the foundation Obra Social La Caixa and the European Research
Council (ERC- StG-757397). J.S.M. and R.R.-T. have support from the CERCA Programme and by the Commission for Universities and Research of the Department of Innovation, Universities, and Enterprise of the Generalitat de Catalunya (2017 SGR 1079). This work was partially funded by the Spanish Ministry of Economy and Competitiveness (MINE$\mathrm{CO}$ ) through the projects MINDS (Proyectos I+D Excelencia + FEDER): TEC2015-70104-P, BIOBOT (Programa Explora Ciencia/Tecnología): TEC2015- 72718-EXP and EuUONANOMED II PCIN-2016-025. R.J.A. thanks the ISRAEL SCIENCE FOUNDATION (Grant No. 1553/18) for the support of this research. The project that gave rise to these results received the support of a fellowship from "la Caixa" Foundation (ID 1000010434). The fellowship code of A.G.M. is $\mathrm{LCF} / \mathrm{BQ} / \mathrm{DI} 17 / 11620054$. This project has received funding from the European Union's Horizon 2020 research and innovation programme under the Marie Skłodowska-Curie Grant Agreement No. 713673.

\section{ABBREVIATIONS}

DDS, drug delivery systems; DMEM, Dulbecco's modified Eagle medium; EB, endothelial barrier; ECs, endothelial cells; ECM, extracellular matrix; EPR, endothelial permeability and retention effect; FRET, Förster resonance energy transfer; HUVECs, human umbilical vein endothelial cells; NPs, nanoparticles; TME, tumor microenvironment

\section{REFERENCES}

(1) Farokhzad, O. C.; Langer, R. Nanomedicine: Developing Smarter Therapeutic and Diagnostic Modalities. Adv. Drug Delivery Rev. 2006, 58 (14), 1456-1459.

(2) Steichen, S. D.; Caldorera-Moore, M.; Peppas, N. A. A Review of Current Nanoparticle and Targeting Moieties for the Delivery of Cancer Therapeutics. Eur. J. Pharm. Sci. 2013, 48 (3), 416-427.

(3) James, N. D.; Coker, R. J.; Tomlinson, D.; Harris, J. R. W.; Gompels, M.; Pinching, A. J.; Stewart, J. S. W. Liposomal Doxorubicin (Doxil): An Effective New Treatment for Kaposi's Sarcoma in AIDS. Clin. Oncol. 1994, 6 (5), 294-296.

(4) Wilhelm, S.; Tavares, A. J.; Dai, Q.; Ohta, S.; Audet, J.; Dvorak, H. F.; Chan, W. C. W. Analysis of Nanoparticle Delivery to Tumours. Nat. Rev. Mater. 2016, 1, 16014.

(5) Jain, R. K.; Stylianopoulos, T. Delivering Nanomedicine to Solid Tumors. Nat. Rev. Clin. Oncol. 2010, 7 (11), 653-664.

(6) Owen, S. C.; Chan, D. P. Y.; Shoichet, M. S. Polymeric Micelle Stability. Nano Today 2012, 7 (1), 53-65.

(7) Holme, M. N.; Fedotenko, I. A.; Abegg, D.; Althaus, J.; Babel, L.; Favarger, F.; Reiter, R.; Tanasescu, R.; Zaffalon, P.-L.; Ziegler, A.; Müller, B.; Saxer, T.; Zumbuehl, A. Shear-Stress Sensitive Lenticular Vesicles for Targeted Drug Delivery. Nat. Nanotechnol. 2012, 7 (8), 536-543.

(8) Shi, Y.; Lammers, T.; Storm, G.; Hennink, W. E. PhysicoChemical Strategies to Enhance Stability and Drug Retention of Polymeric Micelles for Tumor-Targeted Drug Delivery. Macromol. Biosci. 2017, 17 (1), 1600160.

(9) Barua, S.; Mitragotri, S. Challenges Associated with Penetration of Nanoparticles across Cell and Tissue Barriers: A Review of Current Status and Future Prospects. Nano Today 2014, 9 (2), 223-243.

(10) Maeda, H.; Wu, J.; Sawa, T.; Matsumura, Y.; Hori, K. Tumor Vascular Permeability and the EPR Effect in Macromolecular Therapeutics: A Review. J. Controlled Release 2000, 65 (1), 271-284.

(11) Upponi, J. R.; Torchilin, V. P.; Alonso, M. J.; Garcia-Fuentes, M. Passive vs. Active Targeting: An Update of the EPR Role in Drug Delivery to Tumors. Nano-Oncologicals: New Targeting and Delivery Approaches 2014, 3-45, DOI: 10.1007/978-3-319-08084-0_1. 
(12) Golombek, S. K.; May, J.-N.; Theek, B.; Appold, L.; Drude, N.; Kiessling, F.; Lammers, T. Tumor Targeting via EPR: Strategies to Enhance Patient Responses. Adv. Drug Delivery Rev. 2018, 130, 17-38.

(13) Heldin, C.-H.; Rubin, K.; Pietras, K.; Östman, A. High Interstitial Fluid Pressure - an Obstacle in Cancer Therapy. Nat. Rev. Cancer 2004, 4 (10), 806-813.

(14) Chen, H.; Kim, S.; He, W.; Wang, H.; Low, P. S.; Park, K.; Cheng, J.-X. Fast Release of Lipophilic Agents from Circulating PEG-PDLLA Micelles Revealed by in Vivo Förster Resonance Energy Transfer Imaging. Langmuir 2008, 24 (10), 5213-5217.

(15) Lu, J.; Owen, S. C.; Shoichet, M. S. Stability of Self-Assembled Polymeric Micelles in Serum. Macromolecules 2011, 44 (15), 60026008.

(16) Gravier, J.; Sancey, L.; Hirsjärvi, S.; Rustique, E.; Passirani, C.; Benoît, J.-P.; Coll, J.-L.; Texier, I. FRET Imaging Approaches for in Vitro and in Vivo Characterization of Synthetic Lipid Nanoparticles. Mol. Pharmaceutics 2014, 11 (9), 3133-3144.

(17) Aguilar-Castillo, B. A.; Santos, J. L.; Luo, H.; Aguirre-Chagala, Y. E.; Palacios-Hernández, T.; Herrera-Alonso, M. Nanoparticle Stability in Biologically Relevant Media: Influence of Polymer Architecture. Soft Matter 2015, 11 (37), 7296-7307.

(18) Sun, X.; Wang, G.; Zhang, H.; Hu, S.; Liu, X.; Tang, J.; Shen, Y. The Blood Clearance Kinetics and Pathway of Polymeric Micelles in Cancer Drug Delivery. ACS Nano 2018, 12 (6), 6179-6192.

(19) Feiner-Gracia, N.; Buzhor, M.; Fuentes, E.; Pujals, S.; Amir, R. J.; Albertazzi, L. Micellar Stability in Biological Media Dictates Internalization in Living Cells. J. Am. Chem. Soc. 2017, 139 (46), 16677-16687.

(20) Breslin, S.; O'Driscoll, L. Three-Dimensional Cell Culture: The Missing Link in Drug Discovery. Drug Discovery Today 2013, 18 (5), 240-249.

(21) Gupta, N.; Liu, J. R.; Patel, B.; Solomon, D. E.; Vaidya, B.; Gupta, V. Microfluidics-Based 3D Cell Culture Models: Utility in Novel Drug Discovery and Delivery Research. Bioeng. Transl. Med. 2016, 1 (1), 6381.

(22) Jeon, J. S.; Zervantonakis, I. K.; Chung, S.; Kamm, R. D.; Charest, J. L. In Vitro Model of Tumor Cell Extravasation. PLoS One 2013, 8 (2), e56910.

(23) Truong, D.; Puleo, J.; Llave, A.; Mouneimne, G.; Kamm, R. D.; Nikkhah, M. Breast Cancer Cell Invasion into a Three Dimensional Tumor-Stroma Microenvironment. Sci. Rep. 2016, 6, 34094.

(24) Kim, M.-C.; Whisler, J.; Silberberg, Y. R.; Kamm, R. D.; Asada, H. H. Cell Invasion Dynamics into a Three Dimensional Extracellular Matrix Fibre Network. PLoS Comput. Biol. 2015, 11 (10), e1004535.

(25) Kalchman, J.; Fujioka, S.; Chung, S.; Kikkawa, Y.; Mitaka, T.; Kamm, R. D.; Tanishita, K.; Sudo, R. A Three-Dimensional Microfluidic Tumor Cell Migration Assay to Screen the Effect of Anti-Migratory Drugs and Interstitial Flow. Microfluid. Nanofluid. 2013, 14 (6), 969-981.

(26) Jeong, G. S.; Han, S.; Shin, Y.; Kwon, G. H.; Kamm, R. D.; Lee, S.H.; Chung, S. Sprouting Angiogenesis under a Chemical Gradient Regulated by Interactions with an Endothelial Monolayer in a Microfluidic Platform. Anal. Chem. 2011, 83 (22), 8454-8459.

(27) Farahat, W. A.; Wood, L. B.; Zervantonakis, I. K.; Schor, A.; Ong, S.; Neal, D.; Kamm, R. D.; Asada, H. H. Ensemble Analysis of Angiogenic Growth in Three-Dimensional Microfluidic Cell Cultures. PLoS One 2012, 7 (5), e37333.

(28) Ko, J.; Ahn, J.; Kim, S.; Lee, Y.; Lee, J.; Park, D.; Jeon, N. L. Tumor Spheroid-on-a-Chip: A Standardized Microfluidic Culture Platform for Investigating Tumor Angiogenesis. Lab Chip 2019, 19 (17), 2822-2833.

(29) Nashimoto, Y.; Okada, R.; Hanada, S.; Arima, Y.; Nishiyama, K.; Miura, T.; Yokokawa, R. Vascularized Cancer on a Chip: The Effect of Perfusion on Growth and Drug Delivery of Tumor Spheroid. Biomaterials 2020, 229, 119547.

(30) Ho, Y. T.; Adriani, G.; Beyer, S.; Nhan, P.-T.; Kamm, R. D.; Kah, J. C. Y. A Facile Method to Probe the Vascular Permeability of Nanoparticles in Nanomedicine Applications. Sci. Rep. 2017, 7 (1), 707.

(31) Tang, Y.; Soroush, F.; Sheffield, J. B.; Wang, B.; Prabhakarpandian, B.; Kiani, M. F. A Biomimetic Microfluidic Tumor
Microenvironment Platform Mimicking the EPR Effect for Rapid Screening of Drug Delivery Systems. Sci. Rep. 2017, 7 (1), 9359.

(32) Young, E. W. K.; Watson, M. W. L.; Srigunapalan, S.; Wheeler, A. R; Simmons, C. A. Technique for Real-Time Measurements of Endothelial Permeability in a Microfluidic Membrane Chip Using Laser-Induced Fluorescence Detection. Anal. Chem. 2010, 82 (3), $808-816$.

(33) Griffith, L. G.; Swartz, M. A. Capturing Complex 3D Tissue Physiology in Vitro. Nat. Rev. Mol. Cell Biol. 2006, 7 (3), 211-224.

(34) Xu, X.; Farach-Carson, M. C.; Jia, X. Three-Dimensional in Vitro Tumor Models for Cancer Research and Drug Evaluation. Biotechnol. Adv. 2014, 32 (7), 1256-1268.

(35) Ng, C. P.; Pun, S. H. A Perfusable 3D Cell-Matrix Tissue Culture Chamber for in Situ Evaluation of Nanoparticle Vehicle Penetration and Transport. Biotechnol. Bioeng. 2008, 99 (6), 14901501.

(36) Albanese, A.; Lam, A. K.; Sykes, E. A.; Rocheleau, J. V.; Chan, W. C. W. Tumour-on-a-Chip Provides an Optical Window into Nanoparticle Tissue Transport. Nat. Commun. 2013, 4, 2718.

(37) Lazzari, G.; Couvreur, P.; Mura, S. Multicellular Tumor Spheroids: A Relevant 3D Model for the in Vitro Preclinical Investigation of Polymer Nanomedicines. Polym. Chem. 2017, 8 (34), 4947-4969.

(38) Sindhwani, S.; Syed, A. M.; Ngai, J.; Kingston, B. R.; Maiorino, L.; Rothschild, J.; MacMillan, P.; Zhang, Y.; Rajesh, N. U.; Hoang, T.; Wu, J. L. Y.; Wilhelm, S.; Zilman, A.; Gadde, S.; Sulaiman, A.; Ouyang, B.; Lin, Z.; Wang, L.; Egeblad, M.; Chan, W. C. W. The Entry of Nanoparticles into Solid Tumours. Nat. Mater. 2020, 19 (5), 566-575.

(39) Wang, H.-F.; Ran, R.; Liu, Y.; Hui, Y.; Zeng, B.; Chen, D.; Weitz, D. A.; Zhao, C.-X. Tumor-Vasculature-on-a-Chip for Investigating Nanoparticle Extravasation and Tumor Accumulation. ACS Nano 2018, 12 (11), 11600-11609.

(40) Chen, Y.; Gao, D.; Wang, Y.; Lin, S.; Jiang, Y. A Novel 3D BreastCancer-on-Chip Platform for Therapeutic Evaluation of Drug Delivery Systems. Anal. Chim. Acta 2018, 1036, 97-106.

(41) Kwak, B.; Ozcelikkale, A.; Shin, C. S.; Park, K.; Han, B. Simulation of Complex Transport of Nanoparticles around a Tumor Using Tumor-Microenvironment-on-Chip. J. Controlled Release 2014, 194, 157-167.

(42) Ravi, M.; Paramesh, V.; Kaviya, S. R.; Anuradha, E.; Solomon, F. D. P. 3D Cell Culture Systems: Advantages and Applications. J. Cell. Physiol. 2015, 230 (1), 16-26.

(43) Doshi, N.; Prabhakarpandian, B.; Rea-Ramsey, A.; Pant, K.; Sundaram, S.; Mitragotri, S. Flow and Adhesion of Drug Carriers in Blood Vessels Depend on Their Shape: A Study Using Model Synthetic Microvascular Networks. J. Controlled Release 2010, 146 (2), 196-200.

(44) Pampaloni, F.; Reynaud, E. G.; Stelzer, E. H. K. The Third Dimension Bridges the Gap between Cell Culture and Live Tissue. Nat. Rev. Mol. Cell Biol. 2007, 8 (10), 839-845.

(45) Hutmacher, D. W. Biomaterials Offer Cancer Research the Third Dimension. Nat. Mater. 2010, 9 (2), 90-93.

(46) Buzhor, M.; Harnoy, A. J.; Tirosh, E.; Barak, A.; Schwartz, T.; Amir, R. J. Supramolecular Translation of Enzymatically Triggered Disassembly of Micelles into Tunable Fluorescent Responses. Chem. Eur. J. 2015, 21 (44), 15633-15638.

(47) Zervantonakis, I. K.; Hughes-Alford, S. K.; Charest, J. L.; Condeelis, J. S.; Gertler, F. B.; Kamm, R. D. Three-Dimensional Microfluidic Model for Tumor Cell Intravasation and Endothelial Barrier Function. Proc. Natl. Acad. Sci. U. S. A. 2012, 109 (34), 1351513520.

(48) Tornavaca, O.; Chia, M.; Dufton, N.; Almagro, L. O.; Conway, D. E.; Randi, A. M.; Schwartz, M. A.; Matter, K.; Balda, M. S. ZO-1 Controls Endothelial Adherens Junctions, Cell-Cell Tension, Angiogenesis, and Barrier Formation. J. Cell Biol. 2015, 208 (6), 821-838.

(49) Sinha, R.; Le Gac, S.; Verdonschot, N.; van den Berg, A.; Koopman, B.; Rouwkema, J. Endothelial Cell Alignment as a Result of Anisotropic Strain and Flow Induced Shear Stress Combinations. Sci. Rep. 2016, 6, 29510 DOI: 10.1038/srep29510. 
(50) Aird, W. C. Spatial and Temporal Dynamics of the Endothelium.

J. Thromb. Haemostasis 2005, 3 (7), 1392-1406.

(51) Tzima, E.; Irani-Tehrani, M.; Kiosses, W. B.; Dejana, E.; Schultz,

D. A.; Engelhardt, B.; Cao, G.; DeLisser, H.; Schwartz, M. A. A Mechanosensory Complex That Mediates the Endothelial Cell Response to Fluid Shear Stress. Nature 2005, 437 (7057), 426.

(52) Adriani, G.; Ma, D.; Pavesi, A.; Kamm, R. D.; Goh, E. L. K. A 3D Neurovascular Microfluidic Model Consisting of Neurons, Astrocytes and Cerebral Endothelial Cells as a Blood-Brain Barrier. Lab Chip 2017, 17 (3), 448-459.

(53) Booth, R.; Kim, H. Characterization of a Microfluidic in Vitro Model of the Blood-Brain Barrier (MBBB). Lab Chip 2012, 12 (10), 1784.

(54) Herland, A.; van der Meer, A. D.; FitzGerald, E. A.; Park, T.-E.; Sleeboom, J. J. F.; Ingber, D. E. Distinct Contributions of Astrocytes and Pericytes to Neuroinflammation Identified in a 3D Human BloodBrain Barrier on a Chip. PLoS One 2016, 11 (3), e0150360.

(55) Baker, B. M.; Chen, C. S. Deconstructing the Third Dimension: How 3D Culture Microenvironments Alter Cellular Cues. J. Cell Sci. 2012, 125 (13), 3015-3024.

(56) LaBarbera, D. V.; Reid, B. G.; Yoo, B. H. The Multicellular Tumor Spheroid Model for High-Throughput Cancer Drug Discovery. Expert Opin. Drug Discovery 2012, 7 (9), 819-830.

(57) Wilson, W. R.; Hay, M. P. Targeting Hypoxia in Cancer Therapy. Nat. Rev. Cancer 2011, 11 (6), 393-410.

(58) Minamikawa-Tachino, R.; Ogura, K.; Ito, A.; Nagayama, K. Time-Lapse Imaging of HeLa Spheroids in Soft Agar Culture Provides Virtual Inner Proliferative Activity. PLoS One 2020, 15 (4), e0231774.

(59) Pereira, P. M. R.; Berisha, N.; Bhupathiraju, N. V. S. D. K.; Fernandes, R.; Tomé, J. P. C.; Drain, C. M. Cancer Cell Spheroids Are a Better Screen for the Photodynamic Efficiency of Glycosylated Photosensitizers. PLoS One 2017, 12 (5), e0177737.

(60) Tight Junctions, 2nd ed.; Routledge Taylor \& Francis Group, 2001. https://www.crcpress.com/Tight-Junctions/CereijidoAnderson/p/book/9780849323836 (accessed 07-10-2019).

(61) Tarbell, J. M. Shear Stress and the Endothelial Transport Barrier. Cardiovasc. Res. 2010, 87 (2), 320-330.

(62) Kaji, H.; Yokoi, T.; Kawashima, T.; Nishizawa, M. Controlled Cocultures of HeLa Cells and Human Umbilical Vein Endothelial Cells on Detachable Substrates. Lab Chip 2009, 9 (3), 427-432.

(63) Maeda, H. Toward a Full Understanding of the EPR Effect in Primary and Metastatic Tumors as Well as Issues Related to Its Heterogeneity. Adv. Drug Delivery Rev. 2015, 91, 3-6.

(64) Tchoryk, A.; Taresco, V.; Argent, R. H.; Ashford, M.; Gellert, P. R.; Stolnik, S.; Grabowska, A.; Garnett, M. C. Penetration and Uptake of Nanoparticles in 3D Tumor Spheroids. Bioconjugate Chem. 2019, 30 (5), 1371-1384.

(65) Agarwal, R.; Jurney, P.; Raythatha, M.; Singh, V.; Sreenivasan, S. V.; Shi, L.; Roy, K. Effect of Shape, Size, and Aspect Ratio on Nanoparticle Penetration and Distribution inside Solid Tissues Using 3D Spheroid Models. Adv. Healthcare Mater. 2015, 4 (15), 2269-2280.

(66) Lu, H.; Utama, R. H.; Kitiyotsawat, U.; Babiuch, K.; Jiang, Y.; Stenzel, M. H. Enhanced Transcellular Penetration and Drug Delivery by Crosslinked Polymeric Micelles into Pancreatic Multicellular Tumor Spheroids. Biomater. Sci. 2015, 3 (7), 1085-1095.

(67) Du, A. W.; Lu, H.; Stenzel, M. Stabilization of PaclitaxelConjugated Micelles by Cross-Linking with Cystamine Compromises the Antitumor Effects against Two- and Three-Dimensional Tumor Cellular Models. Mol. Pharmaceutics 2016, 13 (11), 3648-3656.

(68) Arranja, A.; Denkova, A. G.; Morawska, K.; Waton, G.; van Vlierberghe, S.; Dubruel, P.; Schosseler, F.; Mendes, E. Interactions of Pluronic Nanocarriers with 2D and 3D Cell Cultures: Effects of PEO Block Length and Aggregation State. J. Controlled Release 2016, 224, 126-135. 